\title{
Interpreting higher computations as types with totality
}

\author{
by \\ Lill Kristiansen \\ Dag Normann \\ Institute of Mathematics \\ The University of Oslo
}

\section{Introduction}

Qualitative domains were introduced by Girard [ 5 ] in order to give a model of system F, originally introduced by Girard [ 2 ]. The closed terms in system $\mathrm{F}$ will be interpreted as consistent objects in their corresponding domains. Girard added a notion of totality to the qualitative domains. A free type variable may be interpreted as any qualitative domain with any set of consistent sets representing the total objects. When the free variables are abstracted away, each closed type-term is interpreted as a specific domain with a specific set of total objects. Since there is no restriction on the families that may be used as the total objects, there is no structural or conceptual analysis of the notion of totality in Girard's work.

A general line of interest to both authors has been the search for a conceptual understanding of totality. The first author has carried out a program for investigation the notion of totality for qualitative domains.

In Normann [ 8 ] the notion of totality is discussed and axiomatized. It is shown that the elements of a fix-point of a strictly positive inductive definition within a reasonable semantics for type theory will satisfy the suggested axioms of totality set up. Berger [1] discusses the notion of totality for general domains and effective totality for effective domains. He uses his notion of totality for generalizing e.g. the Kreisel-LacombeShoenfield theorem to domains.

The basis for this paper is an analysis of totality due to Kristiansen. Kristiansen has isolated a family of structures which will be qualitative domains with a set of total objects. The notion of totality is partly based on the conceptual discussion in [ 8 ]. An object will be total if it "answers" a fixed set of "questions", represented by stable chains. In addition Kristiansen's total objects and questions are effectively dense. This is 
reflected in the supporting E-structures that will be described in section 2. The notion of totality used here is more restricted than that of Berger [ 1 ]. It will for instance not be the case in general that the set of maximal elements may form a set of total objects. This, and other results not used here will appear in the forthcoming Kristiansen [ 7 ].

In this paper we will construct a hierarchy of coherence-spaces (i.e. qualitative domains where consistency is 2-based ) with totality, starting with the natural numbers as the base-type and using infinite dependent products for forming new types. Our main theorem is that the length of this hierarchy corresponds to recursion in the Kleene-functional ${ }^{3} \mathrm{E}$. The proof is an implementation of a general proof-frame, and we expect similar results to hold for other natural ways of modelling types, e.g. using domain theory.

In Normann [ 9] a similar result is shown using the empty type. There is no significant analysis of totality behind the proof in [ 9 ]. The virtue of that proof is that the complexity can be shown using constructions standard in type theory.

In this paper the domains will be effectively nonempty. One main aspect of the proof in this paper is that if we restrict ourselves to a hierarchy of effectively nonempty types, we may ad a fine-structure on the types that is so rich that it enables us to carry out finer recursive constructions. We expect that these methods can be used for investigating other semantics of type-theory as well, and thereby throw some light on the various typeconstructors used. We also see our result as the first developement of tools for investigating sets of high complexity in the same way that the KleeneKreisel continuous functionals, and the Dilators and Ptykes ( Girard [ 3 ] and [ 4$]$ ), can be used to investigate concepts with complexity in the projective or analytic hierarchy.

Finally we think that the main theorem itself is of a nature where its proof will set a standard for the kind of fine-structure we are looking for in the semantics of type-theory.

$2 E$ - structures and totality

We will assume familiarity with the notions of qualitative domains and coherence spaces as introduced in Girard [ 5 ]

\subsection{Definition}

Let $X$ be a qualitative domain.

a) A chain in $X$ will be a set $C$ of pairwise inconsistent finite consistent sets. 
b) If $x \in X$ and $C$ is a chain in $X$, then $x$ meets $C$ if $x$ contains an element of $C$ as a subset.

If $C_{1}$ and $C_{2}$ are chains in $X$ we may form the product $C_{1} \times C_{2}$ by

$$
C_{1} \times C_{2}=\left\{A_{1} \cup A_{2} \in X \mid A_{1} \in C_{1} \text { and } A_{2} \in C_{2}\right\}
$$

$C_{1} \times C_{2}$ is also a chain, and if $x$ meets $C_{1}$ and $C_{2}$ then $x$ also meets $\mathrm{C}_{1} \times \mathrm{C}_{2}$.

\subsection{Definition}

a) Let $X$ be a qualitative domain.

$X$ is organized to a partial E-structure if it is equipped with

i) To each $a \in|X|$ a chain $C_{X, a}$ containing $\{a\}$.

ii) To each finite $\mathrm{A} \in \mathrm{X}$ an extension $\mathrm{E}_{\mathrm{X}, \mathrm{A}} \in \mathrm{X}$ containing $\mathrm{A}$ as a subset.

Let $X_{\mathrm{EXT}}=\left\{E_{X, A} \mid A \in_{\text {fin }} X\right\}$

$X_{\text {ETOT }}=\left\{x \in X \mid x\right.$ meets $C_{X, a}$ for all $\left.a \in|X|\right\}$

b) $X$ is an $E$-structure if in addition all chains $C_{X, a}$ and all extensions meet, i.e. $X_{\mathrm{EXT}} \subseteq X_{\mathrm{ETOT}}$.

\section{Remark}

Using products of chains we construct chains $C_{X, A}$ containing $A$ for all finite $A \in X$, and $X_{E T O T}$ is not changed by adding these chains.

\subsection{Definition}

An $E$ - structure $X$ can be extended to a totality-domain if we add a set of questions $Q_{X} \cup\{a|a \in| X \mid\}$ and a chain $C_{q}$ to each question $q$, such that

$$
\mathrm{X}_{\mathrm{EXT}} \subseteq \mathrm{X}_{\mathrm{TOT}} \subseteq \mathrm{X}_{\mathrm{ETOT}}
$$

where $X_{\text {TOT }}=\left\{x \in X \mid x\right.$ meets all chains $C_{q}$ for $q \in Q_{X}$ and all chains $C_{a}$ for $\left.a \in|X|\right\}$

We will require that $Q_{X}$ is non-empty and that the chain $C_{q}$ is not dummy, i.e. different from $\{\varnothing\}$.

Our notion of totality is in accordance with the discussion in Normann [ 8 ]. The notion of totality in Berger [1] is somewhat more liberal but covers this case. 
The restriction $\mathrm{X}_{\mathrm{EXT}} \subseteq \mathrm{X}_{\mathrm{TOT}} \subseteq \mathrm{X}_{\mathrm{ETOT}}$ will in some cases rule out $\mathrm{X}_{\mathrm{MAX}}$ as a legal choice for $X_{\text {TOT }}$. In Kristiansen [7] it will be shown that there is no $E$ - structure on $X=(\mathbf{N} \rightarrow \mathbf{N}) \rightarrow \mathbf{N}$ with $X_{\text {TOT }}=X_{\text {MAX }}$.

The standard total elements of $(\mathbf{N} \rightarrow \mathbf{N}) \rightarrow \mathbf{N}$ will however satisfy

$$
\mathrm{X}_{\mathrm{EXT}} \subset \mathrm{X}_{\mathrm{TOT}} \subset \mathrm{X}_{\mathrm{ETOT}} \subset \mathrm{X}_{\mathrm{MAX}}
$$

where $\subset$ is proper inclusion. This will be used in section 4 .

Remark

In a totality-domain we will call a chain total if it meets all $x \in X_{\text {TOT }}$. In an $\mathrm{E}$ - structure we will call a chain ext-total if it meets all $\mathrm{x} \in \mathrm{X}_{\mathrm{EXT}}$ and E-total if it meets all $\mathrm{x} \in \mathrm{X}_{\mathrm{ETOT}}$.

By a modified chain product we may define chains

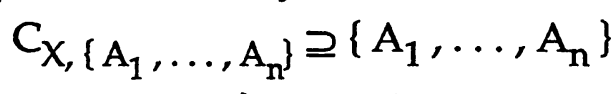

for all finite chains $\left\{A_{1}, \ldots, A_{n}\right\}$ such that $C_{X},\left\{A_{1}, \ldots, A_{n}\right\}$ is an E-total chain.

Girard [ 5 ] uses stable functions for the interpretation of function spaces, and the stable functions are represented by their traces. We assume some familiarity with the use of stable functions, though the original definition can be recovered from definition 2.4 below, using a constant parameterisation.

We see stable functions as a way to model deterministic sequential algorithms, and we will often use such algorithms for proving that a given function is stable.

\subsection{Definition}

a) Let $X$ and $Y$ be two qualitative domains. $X$ is a subdomain of $Y$ if $|X| \subseteq|Y|$, and if $X \subseteq|X|$ then $x \in Y$ if and only if $x \in X$.

b) A stable parameterisation of a family of qualitative domains is a family $\left\{Y_{A}\right\}_{A \in X}$ such that:

i) $\quad c(A)=\left|Y_{A}\right|$ is a stable function, $c: X \rightarrow \mathscr{P}\left(\cup\left\{\left|Y_{A}\right| \mid A \in X\right\}\right.$

ii) If $A_{1} \subseteq A_{2}$ then $Y_{A_{1}}$ is a subdomain of $Y_{A_{2}}$

c) A stable function relative to this parameterisation is an $f: X \rightarrow \cup\left\{Y_{A} \mid A \in X\right\}$ such that

i) $f(A) \in Y_{A}$ ( $f$ gives consistent output)

ii) $A_{1} \subseteq A_{2}$ implies $f\left(A_{1}\right) \subseteq f\left(A_{2}\right)$ ( $f$ is monotone )

iii) If $b \in f(A)$ there is a finite $A^{\prime} \subseteq A$ with $b \in f\left(A^{\prime}\right)$ ( $f$ is finitely based ) 
iv) If $A_{1} \cup A_{2} \in X$ and $b \in f\left(A_{1}\right) \cap f\left(A_{2}\right)$ then $b \in f\left(A_{1} \cap A_{2}\right)$ ( $f$ is uniquely based )

d) The qualitative domain $\Pi(A \in X) Y_{A}$ is defined using the traces of the stable functions:

We let $\left|\Pi(A \in X) Y_{A}\right|=\left\{(A, b) \mid A \in_{\text {fin }} X\right.$ and $\left.b \in\left|Y_{A}\right|\right\}$ $t=\left\{\left(A_{1}, b_{1}\right), \ldots,\left(A_{n}, b_{n}\right)\right\}$ is consistent $\left(\right.$ i.e. $\left.t \in \Pi(A \in X) Y_{A}\right)$ if

i) $A_{i_{1}} \cup \ldots \cup A_{i_{k}}=A \in X \Rightarrow\left\{b_{i_{1}}, \ldots, b_{i_{k}}\right\} \in Y_{A}$

ii) $A_{i} \cup A_{j} \in X \wedge b_{i}=b_{j} \Rightarrow A_{i}=A_{j}$

Then we have the usual bijection between stable functions and $\Pi(A \in X) Y_{A}$ via traces and application. For $t \in \Pi(A \in X) Y_{A}$ the application $f(A)=\lambda A \in X \cdot A p p(t, A)$ is defined by

$$
f(A)=\left\{b \mid\left(\exists A^{\prime} \subseteq A\right)\left(\left(A^{\prime}, b\right) \in t\right)\right\}
$$

The $\Pi$ - construction can be extended to partial E-structures, to Estructures and to totality-domains. Here we will consider parameterisations of partial E-structures and the product of such:

\subsection{Definition}

a) Let $X$ be a qualitative domain.

A stable parameterisation of a family of partial E-structures indexed by $X$ will be a stable parameterisation of the corresponding qualitative domains such that

i) For each $b \in U\left\{\left|Y_{A}\right| \mid A \in X\right\}$, the map $C_{b}(A)=C_{Y_{A}, b}$ is stable (if $b \notin\left|Y_{A}\right|$ then $C_{b}(A)$ will be empty ).

ii) For each finite, consistent $B \subseteq \cup\left\{\left|Y_{A}\right| \mid A \in X\right\}$, the map $E_{B}(A)=E_{Y_{A}, B}$ is stable (if $B \notin Y_{A}$ then $E_{B}(A)$ will be empty).

\subsection{Lemma}

Let $\mathrm{X}$ be a partial E-structure and let $\left\{\mathrm{Y}_{\mathrm{A}}\right\}_{\mathrm{A} \in \mathrm{X}}$ be a stable parameterisation of partial E-structures.

a) $\Pi(A \in X) Y_{A}$ can be organized to a partial E-structure.

b) If $X$ is an E-structure, and $Y_{A^{\prime}}$ is an E-structure for all $A^{\prime} \in X_{E X T}$ then $\Pi(A \in X) Y_{A}$ is an E-structure.

c) If in addition $X$ is a totality-domain and $Y_{A^{\prime}}$ is a totality-domain for all $A^{\prime} \in X_{\text {TOT }}$ then $\Pi(A \in X) Y_{A}$ is a totality-domain. 
Proof

The details of the proof will be worked out in Kristiansen [ 7 ]. Here we will define the chains and the extensions in $\Pi(A \in X) Y_{A}$ and give the intuition behind them. Let $Z=\Pi(A \in X) Y_{A}$.

For part c) $Z_{\mathrm{TOT}}=\left\{f \in Z \mid\left(\forall A \in X_{\mathrm{TOT}}\right)\left(f(A) \in\left(\mathrm{Y}_{\mathrm{A}}\right)_{\mathrm{TOT}}\right\}\right.$.

Let $Q_{Z}=\left\{(A, q) \mid A \in X_{T O T} \wedge q \in Q_{Y_{A}}\right\}$.

The idea is that $(A, q)$ is the question: What will $f(A)$ answer to question $\mathrm{q}$ ?

Of course $Q_{Z}$ is exactly the questions we need to define $Z_{T O T}$, given that $Q_{Y_{A}}$ is the set of questions needed for $\left(Y_{A}\right)_{\text {TOT }}$ for all $A \in X_{\text {TOT }}$.

So we want $C_{(A, q)}$ to meet (the trace of ) $f$ if and only if $f(A)$ meets $C_{\mathrm{q}}$. This is obtained by the following definition:

$C_{(A, q)}$ contains all sets of the form $\left\{\left(A_{1}, b_{1}\right), \ldots,\left(A_{n}, b_{n}\right)\right\}$ where $A_{i}$ is a finite subset of $A$ and $\left\{b_{1}, \ldots, b_{n}\right\} \in C_{q}$.

We use the same construction for producing the chains in the E-structure, i.e. in defining $C_{Z,(A, b)}$ when $(A, b) \in|Z|$ we use $E_{X, A}$ and $C_{Y_{E_{X}, A}}$, as we used $A$ and $C_{q}$ above.

In order to define the extension $E_{Z, t}$ when

$$
t=\operatorname{tr}(g)=\left\{\left(A_{1}, b_{1}\right), \ldots,\left(A_{n}, b_{n}\right)\right\}
$$

we need $C_{X, A_{i}}$ for $i=1, \ldots, n$.

The idea is to have $\operatorname{App}\left(E_{Z, t}, A\right)=E_{Y_{A}, g(A)}$ for all $A \in X_{E X T}$ ( in fact for all $A \in X_{\text {ETOT }}$ ).

Let $E_{Z, t}$ be the trace of the following stable function $\phi$ :

if for some $i, A_{i} \subseteq A$ and $b=b_{i}$ let $b \in \phi(A)$, or if this is not the case

if A meets all chains $C_{X, A_{1}}, \ldots, C_{X, A_{n}}$ (then $g(A)=\left\{b_{i} \mid A_{i} \subseteq A\right\}$ ), then we let $b \in \phi(A)$ if $b \in E_{Y_{A}, g(A)}$.

This proves a).

$C_{Z,\left(A^{\prime}, b\right)}$ tests only $f(A)$ where $A=E_{X, A^{\prime}} \in X_{E X T}$.

Under the assumptions of $b), Y_{A}$ will be an E-structure for $A \in X_{E X T}$

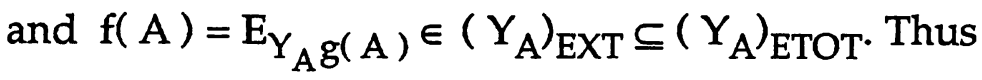

$(\operatorname{tr})(f) \in\left(\Pi(A \in X) Y_{A}\right)_{\text {ETOT }}$ if and only if $f(A) \in\left(Y_{A}\right)_{\text {ETOT }}$ for all $\mathrm{A} \in \mathrm{X}_{\mathrm{EXT}}$.

If $\phi=\lambda A \cdot A p p\left(E_{Z, t}, A\right)$ then $\phi(A) \in\left(Y_{A}\right)_{E X T}$ for all $A \in X_{E T O T}$. 
So when $\mathrm{X}_{\mathrm{EXT}} \subseteq \mathrm{X}_{\mathrm{TOT}} \subseteq \mathrm{X}_{\mathrm{ETOT}}$ and $\left(\mathrm{Y}_{\mathrm{A}}\right)_{\mathrm{EXT}} \subseteq\left(\mathrm{Y}_{\mathrm{A}}\right)_{\mathrm{TOT}} \subseteq\left(\mathrm{Y}_{\mathrm{A}}\right)_{\mathrm{ETOT}}$ for all $A \in X_{\text {TOT }}$ we obtain

$\left(\Pi(A \in X) Y_{A}\right)_{\mathrm{EXT}} \subseteq\left(\Pi(A \in X) Y_{A}\right)_{\mathrm{TOT}} \subseteq\left(\Pi(A \in X) Y_{A}\right)_{\mathrm{ETOT}}$.

This proves the lemma, and establishes $\Pi(A \in X) Y_{A}$ as a totality domain.

We close this section by some constructions needed in section 3 .

\subsection{Definition}

$\mathcal{N}=\{\varnothing,\{n\} \mid n \in \mathbf{N}\}$ with $|\mathcal{N}|=\mathbf{N}$, i.e. the set of natural numbers.

$E_{\mathcal{N}_{0} \varnothing}=\{0\}, E_{\mathcal{N}_{[}\{n\}}=\{n\}, C_{\mathscr{N}_{0} n}=\{\{m\} \mid m \in N\}$ for $n \in N$.

$Q_{\mathcal{X}}$ consists of one question $q$ with $C_{q}=C_{\mathcal{X}}\{n\}$.

This establishes $\mathcal{N}$ as a totality domain.

Similarily we define $\mathcal{N}_{\mathrm{k}}$ as the totality-domain representing $\{0, \ldots, \mathrm{k}\}$. $\mathcal{N}_{k}$ will inherit the E-structure and the totality from $\mathcal{N}_{k}$ so in all respects it will be a substructure of $\mathfrak{N}$.

In Normann [ 8 ] embeddings between totality-domains are discussed and strictly positive inductive definitions are carried out. There are no stability-requirements and nothing like an E-structure is used in [ 8 ]. Moreover we let the empty domain start the induction.

In Kristiansen [ 7 ] we have qualitative domains with E-structure and totality. It is shown that a non-empty least fixpoint of a general positive induction can be interpreted as an E-structure with totality. We exclude the empty domain $\{\varnothing\}$ from being an E-structure with totality.

We do not need embeddings between totality-domains in this paper, only the substructure relation for qualitative domains and the interpretation of $\oplus$ and $\times$ for qualitative domains. These are discussed in detail in Girard [ 5 ], so we will be brief:

\subsection{Definition}

a) Let $X_{1}, \ldots, X_{n}$ be qualitative domains.

We define $X=X_{1} \times \ldots \times X_{n}$ by

$$
|X|=\left(\{1\} \times\left|X_{1}\right|\right) \cup \ldots \cup\left(\{n\} \times\left|X_{n}\right|\right\}
$$

For $x \subseteq|X|$ let $\pi_{i}(x)=\{a \mid(i, a) \in x\}$

Then we let $x \in X$ when $\pi_{i}(x) \in X_{i}$ for all $i=1, \ldots, n$.

We will sometimes write $x=\left(x_{1}, \ldots, x_{n}\right)$ for $x_{i}=\pi_{i}(x)$.

b) If $X_{1}$ and $X_{2}$ are qualitative domains, we define $Y=X_{1} \oplus X_{2}$ by 
$|Y|=\left(\{1\} \times\left|X_{1}\right|\right) \cup\left(\{r\} \times\left|X_{2}\right|\right)$.

If $\mathrm{y} \in|\mathrm{Y}|$ we let $\mathrm{y} \in \mathrm{Y}$ if for some $\mathrm{y}_{1}$ in $Y_{1}$ we have that $\mathrm{y}=\{1\} \times \mathrm{y}_{1}$ or if for some $y_{2}$ in $Y_{2}$ we have that $y=\{r\} \times y_{2}$.

We will sometimes write $r(a)$ for $(r, a)$ and $r(x)$ for $\{r\} \times x$, etc.

In Kristiansen [ 7 ] it will be shown how these constructions can be extended to E-structures and to totality-domains.

3 Codes for syntactic forms

In this section we will define a hierarchy of E-structures with totality with the natural numbers $\mathcal{N}$ at the base and using products of stable parameterisations for obtaining new structures.

We will let all structures be substructures (as domains ) of one universal domain $\mathrm{D}$, and we will represent the various elements of the hierarchy by consistent sets $\mathrm{S}$ in a domain $\mathrm{S}$ of "syntactic forms".

\subsection{Definition}

a) Let $D_{0}=\varnothing, D_{k+1}=\mathcal{N}_{k} \oplus\left(D_{k} \rightarrow D_{k}\right)$, and let $D$ be the limit-structure.

b) Let $S_{0}=\mathcal{N}_{0} \oplus \varnothing, S_{k+1}=\mathcal{N}_{0} \oplus\left(\mathcal{N}_{\varnothing} \times S_{k} \times\left(D_{k} \rightarrow S_{k}\right)\right)$, and let $S$ be the limit.

\section{Remark}

$\mathrm{D}$ will be a qualitative domain satisfying

$$
\mathrm{D}=\mathfrak{N} \oplus(\mathrm{D} \rightarrow \mathrm{D})
$$

while $S$ will be a qualitative domain satisfying

$$
S=\mathcal{N}_{0} \oplus\left(\mathcal{N}_{0} \times S \times(D \rightarrow S)\right)
$$

If $s \in S, s \neq \varnothing$ and $s \neq r\left(\varnothing, s_{1}, p\right)$ we have

i) $s=1(\{0\})$

or

ii) $\mathrm{s}=\mathrm{r}\left(\{0\}, \mathrm{s}_{1}, \mathrm{p}\right\}$

The idea is that in case i) $s$ will represent $\mathcal{N}$ while in case ii), if $s_{1}$ represents $X$ and $p(A)$ represents $Y_{A}$ for $A \in X$ then $s$ represents $\Pi(A \in X) Y_{A}$.

Following this idea we will define the interpretation $\mathrm{I}(\mathrm{s})$ for $\mathrm{s} \in \mathrm{S}$ as qualitative domains such that $\{\mathrm{I}(\mathrm{s})\}_{\mathrm{s} \in \mathrm{S}}$ is a stable parameterisation of 
qualitative domains. Also, each $\mathrm{I}(\mathrm{s})$ will be given a partial E-structure, and we will show that $\{I(s)\}_{s \in S}$ also is a stable parameterisation of partial E-structures.

In case i) $\varnothing \in \mathcal{X}$ and $E_{s, \varnothing}=\{0\}$, while in case ii) $\varnothing \in \Pi(A \in X) Y_{A}$ and $\mathrm{E}_{\mathrm{s}, \varnothing}=(\operatorname{tr})\left(\lambda \mathrm{A} \in \mathrm{X} \cdot \mathrm{E}_{\mathrm{Y}_{\mathrm{A}}, \varnothing}\right)$

In order to make $E_{s, \varnothing}$ stable in $s$ it is convenient to use $r(\{0\}, \varnothing, \varnothing)$ as the unique basis for going to the right.

We will use the following conventions: $d$ will be an element in $|D|$ while $\delta$ will be a finite element in $D$.

$s$ will be an arbitrary element of $S$ while $\sigma$ will be a finite element of $S$.

$p$ will be an element of $D \rightarrow S$ while $\pi$ will be a finite element of $D \rightarrow S$.

\subsection{Definition}

We define $\mathrm{I}(\mathrm{s})$ for $s \in \mathrm{S}$ by defining $\mathrm{I}(\sigma)$ for finite $\sigma \in \mathrm{S}$ essentially by induction on the least $\mathrm{k}$ for which $\sigma \in \mathrm{S}_{\mathrm{k}}$.

For $\sigma=\varnothing$, let $|\mathrm{I}(\sigma)|=\varnothing$.

For $\sigma=1(\{0\})$, let $\mathrm{I}(\sigma)=\mathfrak{N} \oplus \varnothing$

If $\sigma=\mathrm{r}\left(\{0\}, \sigma_{1}, \pi\right) \in \mathrm{S}_{\mathrm{k}+1}$, then $\sigma_{1} \in \mathrm{S}_{\mathrm{k}}$ and $\pi \in \mathrm{D}_{\mathrm{k}} \rightarrow \mathrm{S}_{\mathrm{k}}$, and we let $\mathrm{d}=\mathrm{r}\left(\delta_{1}, \mathrm{~d}_{2}\right) \in|\mathrm{I}(\sigma)|$ if and only if $\delta_{1} \in \mathrm{I}\left(\sigma_{1}\right)$ and $\mathrm{d}_{2} \in \mid \mathrm{I}\left(\pi\left(\delta_{1}\right) \mid\right.$.

Consistency is inherited from $D$, i.e. consistency of traces.

\subsection{Lemma}

The function $c(s)=|I(s)|$ is a stable function from $S$ to $\mathscr{P}(|D|)$.

Proof

By induction on $\mathrm{k}$ we can prove this for $\sigma \in \mathrm{S}_{\mathrm{k}}$. The details are left for the reader.

We see that $\mathrm{I}(\mathrm{s})$ is the minimal solution to the equation system

* $\mathrm{I}(\mathrm{I}(\{0\})=\mathcal{N} \oplus \varnothing$

** I( $\mathrm{r}(\{0\}, \mathrm{s}, \mathrm{p}))=\varnothing \oplus \Pi(\mathrm{x} \in \mathrm{I}(\mathrm{s})) \mathrm{I}(\mathrm{p}(\mathrm{s}))$

and lemma 3.3 ensures that this minimal solution exists.

Likewise we can use the construction of lemma 2.6 to organize each $\mathrm{I}(\mathrm{s})$ to a partial E-structure. By induction on $k$ we can prove that for $\sigma \in S_{k}$ this is a stable parameterisation, and thus $\{\mathrm{I}(\mathrm{s})\}_{\mathrm{s} \in \mathrm{S}}$ can be considered as 
a stable parameterisation of partial E-structures. We thus have

\subsection{Theorem}

There is a minimal solution $\{\mathrm{I}(\mathrm{s})\}_{\mathrm{s} \in \mathrm{S}}$ as a stable parameterisation of partial E-structures such that the equations ${ }^{*}$ and ${ }^{* *}$ hold.

We will now define our hierarchy of types, $\{\mathrm{I}(\mathrm{s})\}_{\mathrm{s} \in \mathrm{S}_{\mathrm{wf}}}$ :

\subsection{Definition}

Simultanously we define $\mathrm{S}_{\mathrm{wf}} \subseteq \mathrm{S}$ and $(\mathrm{I}(\mathrm{s}))_{\mathrm{TOT}}$ for $\mathrm{s} \in \mathrm{S}_{\mathrm{wf}}$ as the least solution of

i) $l(\{0\})\} \in S_{w f}$ with $(\mathrm{I}(\mathrm{s}))_{\mathrm{TOT}}$ as $\{\mathrm{l}(\{\mathrm{n}\}) \mid \mathrm{n} \in \mathbf{N}\}$

ii) $\mathrm{r}(\{0\}, \mathrm{s}, \mathrm{p}\} \in \mathrm{S}_{\mathrm{wf}}$ if $\mathrm{s} \in \mathrm{S}_{\mathrm{wf}}$ and for all $\mathrm{x} \in(\mathrm{I}(\mathrm{s}))_{\mathrm{TOT}}$ we have that $\mathrm{p}(\mathrm{x}) \in \mathrm{S}_{\mathrm{wf}}$.

$(\mathrm{I}(\mathrm{r}(\{0\}, \mathrm{s}, \mathrm{p}\}))$ TOT will be $\mathrm{r}(\{\mathrm{x} \mid \mathrm{x}$ is the trace of a stable function mapping an element $\mathrm{y} \in(\mathrm{I}(\mathrm{s}))_{\mathrm{TOT}}$ onto an element of $\left.(\mathrm{I}(\mathrm{p}(\mathrm{y})))_{\text {TÓт }}\right\}$

Using lemma $2.6 \mathrm{c}$ ), lemma 3.4 and induction we see that when $s \in \mathrm{S}_{\mathrm{wf}}$ then $I(s)$ is a totality-domain, and thus $S_{w f}$ is well defined.

In section 4 we will investigate the length of this inductive definition. In order to do so we need some more structure on the domains $I(s)$ for $s \in \mathrm{S}_{\mathrm{wf}}$. Notice that if $s \in \mathrm{S}_{\mathrm{wf}}$ then all extensions in $\mathrm{I}(\mathrm{s})$ are total and the total objects meet all the chains.

\subsection{Definition}

Assume that we have a fixed enumeration of each finite $\delta \in D$.

We define the stable functions $\mathrm{I}^{\mathrm{d}}: \mathrm{S} \rightarrow \mathcal{P}(|\mathrm{D}|)$ and $\mathrm{I}_{\mathrm{f}}^{\mathrm{d}} \mathrm{S} \rightarrow \mathcal{P}\left(|\mathrm{D}|^{<\omega}\right)$

as the minimal solution to the following equations:

$\mathrm{Id}^{\mathrm{d}}(\mathrm{I}(\{0\}))=|\varnothing \oplus(\mathrm{D} \rightarrow \mathrm{D})|$

$\mathrm{I}^{\mathrm{d}}(\mathrm{r}(\{0\}, \mathrm{s}, \mathrm{p})=|\mathcal{N} \oplus \varnothing|$

$$
\begin{aligned}
& \cup\left\{\mathrm{r}(\delta, \mathrm{d}) \mid \delta \in \mathrm{Id}_{\mathrm{f}}(\mathrm{s})\right\} \\
& \cup\left\{\mathrm{r}(\delta, \mathrm{d})|\delta \subseteq| \mathrm{I}(\mathrm{s}) \mid \wedge\left(\mathrm { d } \in \mathrm { I } ^ { \mathrm { d } } ( \mathrm { p } ( \mathrm { E } _ { \mathrm { I } ( \mathrm { s } ) , \delta } ) ) \vee \mathrm { d } \in \mathrm { I } \left(\mathrm{p}\left(\mathrm{E}_{\mathrm{I}(\mathrm{s}), \delta}\right)\right.\right.\right.
\end{aligned}
$$

but the basis for this $\left(\subseteq \mathrm{E}_{\mathrm{I}(\mathrm{s}), \delta}\right)$ is not contained in $\left.\delta\right\}$.

$\mathrm{Id}_{\mathrm{f}}(\mathrm{s})=\left\{\delta=\left\{\mathrm{d}_{1}, \ldots, \mathrm{d}_{\mathrm{n}}\right\} \in \mathrm{D} \mid \mathrm{n} \geq 1\right.$ and $\exists \mathrm{k} \leq \mathrm{n}\left(\mathrm{d}_{1} \in|\mathrm{I}(\mathrm{s})| \wedge \ldots \wedge\right.$

$d_{k-1} \in|I(s)| \wedge d_{k} \in I^{d}(s)$. 


\subsection{Lemma}

a) For all $s \in S I^{d}(\sigma) \cap|I(s)|=\varnothing$

$$
\mathrm{I}_{\mathrm{f}}^{\mathrm{d}}(\mathrm{s}) \cap \mathrm{I}(\mathrm{s})=\varnothing
$$

b) For all $s \in S_{w f}$, for all $d \in|D|$ and for all finite $\delta \in D$ we have

$$
\begin{aligned}
& \mathrm{d} \in|\mathrm{I}(\mathrm{s})| \text { or } \mathrm{d} \in \mathrm{I}^{\mathrm{d}}(\mathrm{s}) \\
& \delta \in \mathrm{I}(\mathrm{s}) \text { or } \delta \in \mathrm{I}_{\mathrm{f}}^{\mathrm{d}}(\mathrm{s})
\end{aligned}
$$

The proof is straightforward and is left for the reader.

Notice that we need $3.7 \mathrm{a}$ ) in order to show that $\mathrm{I}^{\mathrm{d}}$ and $\mathrm{I}_{\mathrm{f}}^{\mathrm{d}}$ are stable functions.

The definition of $\mathrm{I}_{\mathrm{f}}^{\mathrm{d}}$ illustrates that stable functions correspond to deterministic, sequential algorithms. If we instead define $\mathrm{I}_{\mathrm{f}}^{\mathrm{d}}$ by

$$
\mathrm{I}_{\mathrm{f}}(\mathrm{s})=\left\{\delta \in \mathrm{D} \mid \exists \mathrm{d} \in \delta\left(\mathrm{d} \in \mathrm{I}^{\mathrm{d}}(\mathrm{s})\right\}\right.
$$

then $\mathrm{I}_{\mathrm{f}}^{\mathrm{d}}$ ( and hence $\mathrm{I}^{\mathrm{d}}$ at the next level) will not be stable.

We will use $|\mathrm{I}(\mathrm{s})|$ and $\mathrm{I}^{\mathrm{d}}(\mathrm{s})$ to transform total elements $\mathrm{x}$ in $\mathrm{I}(\mathrm{s})$ for $s \in \mathrm{S}_{\mathrm{wf}}$ to total functions $h_{\mathrm{x}, \mathrm{s}}$ from $\mathbf{N}$ to $\mathbf{N}$ in a stable way

\subsection{Definition}

Let $\left\{d_{i}\right\}_{i \in N}$ be a fixed enumeration of $|D|$, and let $\left\{\delta_{i}\right\}_{i \in N}$ be a fixed enumeration of the finite elements in $\mathrm{D}$.

For $s \in S$ and $x \in I(s)$ let

$$
h_{x, s}(\{i\})=\left\{\begin{array}{l}
\{0\} \text { if } d_{i} \in I^{d}(s) \\
\{j+1\} \text { if } d_{i} \in|I(s)| \text { and } \delta_{j} \text { is where } x \text { meets } \\
C_{I(s), d_{i}} .
\end{array}\right.
$$

Thus $h_{x, s}(i)$ tests if $d_{i}$ is in $|I(s)|$, and in case it is, find where $x$ meets $C_{I(s), d_{i}}$. So, if $s \in S$ is such that $|I(s)|$ and $I^{d}(s)$ are complementary, we have that $h_{x, s}$ is total if and only if $x \in I(s)_{\text {ETOT }}$.

\subsection{Lemma}

a) $\lambda s \in \mathrm{S} . \lambda \mathrm{x} \in \mathrm{I}(\mathrm{s}) \cdot \mathrm{h}_{\mathrm{x}, \mathrm{s}}$ is stable

b) For $s \in S_{w f}$ and $x, y \in(I(s))_{\text {TOT }}$ we have that $h_{x, s}$ is total and $x=y$ if and only if $h_{x, s}=h_{y, s}$

c) For any $s \in S$ and $x \in I(s)$, if $h_{x, s}$ is total, then $x$ is maximal. 
The proofs are easy and left for the reader. For $c)$, notice that it is a general fact for E-structures $X$ that if $x \in X_{\text {ETOT }}$ then $x \in X_{M A X}$.

4 Simulating recursion in ${ }^{3} E$

We will now prove that the hierarchy $\mathrm{S}_{\mathrm{wf}}$ is as complex as recursion in ${ }^{3} \mathrm{E} .{ }^{3} \mathrm{E}$ is the type- 3 functional defined as follows:

${ }^{3} \mathrm{E}(\mathrm{F})=\left\{\begin{array}{l}0 \text { if } \mathrm{F} \text { is total with constant value } 0 \\ 1 \text { if } \mathrm{F} \text { is total, but not constant } 0\end{array}\right.$

Kleene [ 6] defined a notion of computations $\{e\}\left(\psi_{1}, \ldots, \psi_{k}\right) \approx n$ where $\psi_{1}, \ldots, \psi_{\mathrm{k}}$ is a sequence of total functionals of finite, pure type. We will be interested in computations with input ${ }^{3} \mathrm{E}$ and a sequence $f$ from $\mathbf{N} \cup \mathbf{N}^{\mathbf{N}}$. We will omit the mentioning of ${ }^{3} \mathrm{E}$ in our computations. When we write

$$
\{e\}\{f) \approx n
$$

we mean that $f=f_{1} f_{2}$ and that

$$
\{e\}\left(f_{1},{ }^{3} E, f_{2}\right) \approx n
$$

according to Kleene's original definition.

We will systematically omit the Kleene-scheme S5 in our treatment, since this scheme can be reduced to the other schemes.

We will prove the following theorem

\subsection{Theorem}

Assume that $\{e\}\{f) \downarrow$, i.e. takes a value.

Uniformly in $e, f$ and $n$ there is

1. An element $C(e, f, n) \in S_{w f}$

2. An object $\alpha(e, f, n) \in I(C(e, f, n))$ such that

$$
\alpha(e, f, n) \text { is total in } I(C(e, f, n)) \text { if and only if }\{e\}(f) \approx n \text {. }
$$

With our conventions, this shows that semirecursion in ${ }^{3} \mathrm{E}$ can be reduced to

$$
\left\{(s, x) \mid s \in S_{w f} \text { and } x \text { is total in } I(s)\right\} .
$$

The converse is not hard to prove, see Normann[ 9 ] for an analogue result.

In a way we will construct $C(e, f, n)$ and $\alpha(e, f, n)$ by recursion on the 
computation $\{e\}(f)$. In the case of composition we will use a mollification-technique in order to avoid explicit use of the intermediate value, and for this we will need approximal computations. The approximations will be more at the syntactical than at the computational level, but this will be sufficient for our purposes.

\subsection{Definition}

By recursion on $k$ we define $\{e\}_{k}(f)$ for all $e$ and $f$.

i) $\{e\}_{0}(f)=0$ for all $e, f$.

ii) Assume that $\{e\}_{k}(f)$ is defined for all $e, f$.

We define $\{e\}_{k+1}(f)$ following S1 - S4, S6-S9 as follows

1. $\{e\}(x, f)=x+1:\{e\}_{k+1}(x, f)=x+1$

2. $\{e\}(f)=q:\{e\}_{k+1}(f)=q$

3. $\{$ e $\}(x, f)=x:\{e\}_{k+1}(x, f)=x$

4. $\{e\}(f) \approx\left\{e_{1}\right\}\left(\left\{e_{2}\right\}(f), f\right):\{e\}_{k+1}(f)=\left\{e_{1}\right\}_{k}\left(\left\{e_{2}\right\}_{k}(f), f\right)$

6. $\{e\}(f) \approx\left\{e_{1}\right\}(\sigma(f)):\{e\}_{k+1}(f)=\left\{e_{1}\right\}_{k}(\sigma(f))$

7. $\{e\}(x, f, f)=f(x):\{e\}_{k+1}(x, f, f)=f(x)$

8. $\{e\}(f) \approx{ }^{3} E\left(\lambda g \cdot\left\{e_{1}\right\}(g, f)\right):\{e\}_{k+1}(f)={ }^{3} E\left(\lambda g \cdot\left\{e_{1}\right\}_{k}(g, f)\right)$

9. $\{e\}\left(e_{1}, f\right) \approx\left\{e_{1}\right\}(f):\{e\}_{k+1}\left(e_{1}, f\right)=\left\{e_{1}\right\}_{k}(f)$

Otherwise we let $\{e\}_{k+1}(f)=0$

\subsection{Lemma}

Uniformly in the signature for $\mathbf{f}$ there is a primitive recursive function $\rho$ such that

i) $\{e\}_{k}(f)=\{\rho(k, e)\}(f)$ for all $e, f$.

ii) If $k_{1} \leq k_{2}$ then

$$
\begin{aligned}
& \rho\left(k_{1}, \rho\left(k_{2}, e\right)\right)=\rho\left(k_{1}, e\right) \\
& \rho\left(k_{2}, \rho\left(k_{1}, e\right)\right)=\rho\left(k_{1}, e\right)
\end{aligned}
$$

The proof is trivial and is left for the reader. We will omit any reference to the signature of $f$, though we need it to distinguish between the otherwise-case and the rest.

The next definition represents the main construction in this paper, and the central idea of proof is laid down in this definition:

\subsection{Definition}

By induction on $k$ we define

$$
C_{k}(e, f, n) \text { and } \alpha_{k}(e, f, n)
$$

for all $f$ and $n$. 
We will have that $C_{k}(e, f, n) \in S$ and that the definition is monotone in k.

We will have that $\alpha_{k}(e, f, n) \in I\left(C_{k}(e, f, n)\right)$ and that $\alpha_{k}$ is monotone in $\mathrm{k}$.

Both $C_{k}(e, f, n)$ and $\alpha_{k}(e, f, n)$ are uniformly recursive in $e, f, n$ as subsets of $|S|$ and $|D|$ respectively.

If $\{e\}(f)$ is an initial computation, let $m=\{e\}(f)$.

Let $C_{k}(e, f, n)$ be the element of $S$ representing $(\mathbf{N} \rightarrow \mathbf{N}) \rightarrow \mathbf{N}$ for all k.

Let $\alpha(e, f, n)$ be the element of $I\left(C_{k}(e, f, n)\right)$ representing $\lambda f . \mu k((f(0)=k \wedge n=m) \vee f(k) \neq k)$, and let $\alpha_{k}(e, f, n)=\alpha(e, f, n) \cap\left|D_{k}\right|$.

If $\{e\}(f)$ is at first sight not a computation, we let $C_{k}(e, f, n)$ represent $(\mathbf{N} \rightarrow \mathbf{N}) \rightarrow \mathbf{N}$ for all $k$.

Let $\alpha(e, f, n)$ be the element of $I\left(C_{k}(e, f, n)\right)$ representing $\lambda f . \mu k(f(k) \neq k)$, and let $\alpha_{k}(e, f, n)=\alpha(e, f, n) \cap\left|D_{k}\right|$.

This leaves us with the cases $\mathrm{S} 4, \mathrm{~S} 6, \mathrm{~S} 8$ and $\mathrm{S9}$.

Then we let $C_{0}(e, f, n)=\varnothing$ and $\alpha_{0}(e, f, n)=\varnothing$, and we will define $\mathrm{C}_{\mathrm{k}+1}$ and $\alpha_{\mathrm{k}+1}$ for these cases.

S4: $\quad\{e\}(f) \approx\left\{e_{1}\right\}\left(\left\{e_{2}\right\}(f), f\right)$.

We want $C(e, f, n)$ to represent the following object:

$$
\Pi(m \in N) \Pi\left(x \in I \left(C\left(e_{2}, f, m\right) I(D(x, m))\right.\right.
$$

where $D(x, m)=C\left(e_{1}, m, f, n\right)$ if $x=\alpha\left(e_{2}, f, m\right)$

$D(x, m)=C\left(\rho\left(k, e_{1}\right), m f, n\right)$ for the least $k$ such that

$$
h_{x, C}\left(e_{2}, f, m\right)(k) \neq h_{\alpha\left(e_{2}, f, m\right), C\left(e_{2}, f, m\right)}(k)
$$
when $x \neq \alpha\left(e_{2}, f, m\right)$.

We let $C_{k+1}(e, f, n)$ be the trace of the following function in $m$ and $x$ : $r \in D_{k}(x, m)$ if there is a minimal $k_{1} \leq k$ such that

i) $r \in C_{k_{1}}\left(e_{1}, m, f, n\right)$

or 

ii) $h_{x, C_{k}\left(e_{2}, f, m\right)}\left(k_{1}\right) \neq h_{\alpha_{k}\left(e_{2}, f, m\right), C_{k}\left(e_{2}, f, m\right)}\left(k_{1}\right)$,

and in case ii), $r \in C_{k}\left(\rho\left(k_{1}, e_{1}\right), m, f, n\right)$.

In order to prove that this is stable, we must show that

$$
\mathrm{k}_{1} \leq \mathrm{k}_{2} \Rightarrow \mathrm{C}_{\mathrm{k}_{1}}(\mathrm{e}, \mathrm{f}, \mathrm{n}) \subseteq \mathrm{C}_{\mathrm{k}_{2}}\left(\rho\left(\mathrm{k}_{2}, \mathrm{e}\right), \mathrm{f}, \mathrm{n}\right) .
$$

We will do so when the full definition of $C_{k+1}$ is given.

We also want $\alpha(e, f, n)$ to represent the function

$\lambda \mathrm{m} \cdot \lambda \mathrm{x} \in \mathrm{I}\left(\mathrm{C}\left(\mathrm{e}_{2}, \mathrm{f}, \mathrm{m}\right)\right) \cdot \beta(\mathrm{x}, \mathrm{m})$

where

$$
\begin{aligned}
& \beta(x, m)=\alpha\left(e_{1}, m, f, n\right) \text { if } x=\alpha\left(e_{2}, f, m\right) \\
& \beta(x, m)=E_{C\left(\rho\left(k, e_{1}\right), m, f, n\right), \alpha_{k}\left(e_{1}, m, f, n\right)} \text { for the least } k \text { such that } \\
& h_{x}, C\left(e_{2}, f, m\right)(k) \neq h_{\alpha\left(e_{2}, f, m\right), C\left(e_{2}, f, m\right)}(k) \\
& \quad \text { when } x \neq \alpha\left(e_{2}, f, m\right) .
\end{aligned}
$$

Let $\phi=\lambda m . \lambda x \in I\left(C_{k}\left(e_{2}, f, m\right)\right) \beta_{k}(x, m)$

where we have the following algorithm for computing $d \in \beta_{k}(x, m)$ :

Find a minimal $k_{1} \leq k$ such that

i) $d \in \alpha_{k_{1}}\left(e_{1}, m, f, n\right)$

or

ii) $h_{x}, c_{k}\left(e_{2}, f, m\right)\left(k_{1}\right) \neq h_{\alpha_{k}\left(e_{2}, f, m\right), c_{k}\left(e_{2}, f, m\right)}\left(k_{1}\right)$.

If i) applies, we let $d \in \beta_{k}(x, m)$.

If ii) applies we let $d \in \beta_{k}(x, m)$ if

$d \in E_{C_{k}}\left(\rho\left(k, e_{1}\right), m, f, n\right), \alpha_{k_{1}}\left(e_{1}, m, f, n\right)$

We let $\alpha_{k+1}(e, f, n)$ be the part of $\phi$ that is in $D_{k+1}$.

This end case 4 .

S6: $\quad\{e\}(f) \approx\left\{e_{1}\right\}(\sigma(f))$

Let $\quad C_{k+1}(e, f, n)=C_{k}\left(e_{1}, \sigma(f), n\right)$

$$
\alpha_{k+1}(e, f, n)=\alpha_{k}\left(e_{1}, \sigma(f), n\right)
$$

S8: $\quad\{e\}(f) \approx{ }^{3} E\left(\lambda g .\left\{e_{1}\right\}(g, f)\right)$

We want $C(e, f, 0)$ to represent $\Pi(g \in \mathcal{N} \rightarrow \mathcal{N}) I\left(C\left(e_{1}, g, f, 0\right)\right)$ and $\alpha(e, f, 0)$ to represent $\lambda g . \alpha\left(e_{1}, g, f, 0\right)$. 
Moreover we want $\mathrm{C}(\mathrm{e}, \mathrm{f}, 1)$ to represent $\mathrm{I}(\mathrm{C}(\mathrm{e}, \mathrm{f}, 0)) \rightarrow \mathcal{N}$ and $\alpha(e, f, 1)$ to represent $\lambda x . \mu k\left(h_{x, C(e, f, o)}(k) \neq h_{\alpha(e, f, 0), C(e, f, 0)}(k)\right)$. We do this by letting

$$
\begin{aligned}
& C_{k+1}(e, f, 0) \text { represent } \Pi(g \in \mathcal{N} \rightarrow \mathcal{N}) I\left(C_{k}\left(e_{1}, g, f, 0\right)\right. \\
& \alpha_{k+1}(e, f, 0) \text { represent } \lambda g \cdot \alpha_{k}\left(e_{1}, g, f, 0\right) \text { restricted to } D_{k+1} \\
& C_{k+1}(e, f, 1) \text { represent } I\left(C_{k+1}(e, f, 0)\right) \rightarrow \mathcal{N} \\
& \alpha_{k+1}(e, f, 1) \text { represent } \lambda x . \mu k_{1} \leq k\left(h_{x,} C_{k+1}(e, f, o)(k) \neq\right. \\
& \left.\quad h_{\alpha_{k+1}(e, f, 0), C_{k+1}(e, f, 0)}(k)\right) \text { restricted to } D_{k+1} .
\end{aligned}
$$

If $\mathrm{n}>1$ we let $\mathrm{C}_{\mathrm{k}+1}(\mathrm{e}, \mathrm{f}, \mathrm{n})$ represent $(\mathcal{N} \rightarrow \mathcal{N}) \rightarrow \mathcal{N}$ and we let $\alpha_{k+1}(e, f, n)$ represent $\lambda g \cdot \mu k_{1}\left(g\left(k_{1}\right) \neq k_{1}\right)$ restricted to $D_{k+1}$.

S9: $\quad\{e\}\left(e_{1}, f\right) \approx\left\{e_{1}\right\}(f)$ :

Let $\quad C_{k+1}\left(e, e_{1}, f, n\right)=C_{k}\left(e_{1}, f, n\right)$

$$
\alpha_{k+1}\left(e, e_{1}, f, n\right)=\alpha_{k}\left(e_{1}, f, n\right)
$$

This ends Definition 4.4.

\subsection{Lemma}

a) If $k_{1}<k$ then for any $e, f, n$

$$
\begin{array}{ll}
C_{k_{1}}(e, f, n)=C_{k_{1}}(\rho(k, e), f, n) \\
\alpha_{k_{1}}(e, f, n)=\alpha_{k_{1}}(\rho(k, e), f, n) \\
\text { b) } \text { For all } e, f, n \text { and } k \\
C_{k}(e, f, n) \subseteq C_{k}(\rho(k, e), f, n) \\
\alpha_{k}(e, f, n) \subseteq \alpha_{k}(\rho(k, e), f, n)
\end{array}
$$

\section{Proof}

The proof of a) is by induction on $k_{1}$ while the proof of $b$ ) is by induction on $k$. Both proofs are tedious but simple.

This lemma shows that the definition of $C_{k}$ and $\alpha_{k}$ is sound. Moreover, they are recursive, so they are stable in the variables $e, f, n$.

We obviously have that $\alpha_{k}(e, f, n)$ is finite and that the definitions are monotone in $\mathrm{k}$.

As indicated, we let

$$
\begin{aligned}
& C(e, f, n)=\cup(k \in N) C_{k}(e, f, n) \\
& \alpha(e, f, n)=\cup(k \in N) \alpha_{k}(e, f, n)
\end{aligned}
$$


We complete the proof of Theorem 4.1 by showing:

\subsection{Lemma}

If $\{e\}(f) \downarrow$ then for all $n$

a) $\mathrm{C}(\mathrm{e}, \mathrm{f}, \mathrm{n}) \in \mathrm{S}_{\mathrm{wf}}$ and $\alpha(e, f, n)$ is total in $I(C(e, f, n))$ if and only if $\{e\}(f)=n$

b) $h_{\alpha(e, f, n), C(e, f, n)}$ is total

c) $\alpha(e, f, n)$ is not an extension in $I(C(e, f, n))$, i.e. not one of the $\mathrm{E}_{\mathrm{X}, \delta}$ sets.

\section{Proof}

The lemma is proved by induction on the length of the computation, which gives 8 cases S1-S4, S6-S9. To be formally correct we should first prove the lemma for computations on the form $\{\rho(k, e)\}(f)$ by induction on $k$, but since this will only lead to a repetition of details, we leave it for the reader.

For initial computations, a) is trivial, and in these cases $C$ and $\alpha$ are carefully defined for b) and c) to hold.

The cases S6 and S9 are trivial, so we concentrate on cases S4 and S8.

S4

$$
\{e\}(f)=\left\{e_{1}\right\}\left(\left\{e_{2}\right\}(f), f\right) \text {. }
$$

Let $m_{0}=\left\{e_{2}\right\}(f), n_{0}=\left\{e_{1}\right\}\left(m_{0}, f\right)$

By construction

$$
\mathrm{I}(\mathrm{C}(\mathrm{e}, \mathrm{f}, \mathrm{n}))=\mathrm{r}\left(\Pi(\mathrm{m} \in \mathcal{N}) \Pi\left(x \in I\left(C\left(e_{2}, f, m\right)\right)\right) \mathrm{I}(\mathrm{D}(\mathrm{x}, \mathrm{m}))\right)
$$
where

$$
\begin{aligned}
& D(x, m)=C\left(e_{1}, m, f, n\right) \text { if } x=\alpha\left(e_{2}, f, m\right) \\
& D(x, m)=C\left(\rho\left(k, e_{1}\right), m, f, n\right) \text { for some k otherwise. }
\end{aligned}
$$

Here we use that $h_{\alpha\left(e_{2}, f, m\right), C\left(e_{2}, f, m\right)}$ is total.

If $\mathrm{m} \neq \mathrm{m}_{0}$ and $\mathrm{x}$ is total in $\mathrm{I}\left(\mathrm{C}\left(\mathrm{e}_{2}, \mathrm{f}, \mathrm{m}\right)\right)$ we cannot have that $x=\alpha\left(e_{2}, f, m\right)$, so $h_{x}, C\left(e_{2}, f, m\right) \neq h_{\alpha\left(e_{2}, f, m\right), C\left(e_{2}, f, m\right) \text {, and } D(x, m)}$ $=C\left(\rho\left(k, e_{1}\right), f, m\right)$ for some $k$ depending on $x$. Then $\Pi\left(x \in I\left(C\left(e_{2}, f, m\right)\right)\right) I\left(D(x, m)\right.$ can be represented in $S_{w f}$. Moreover $\alpha(e, f, n)(m)(x)$ is an extension and thus total whenever $x$ is total in $\mathrm{I}\left(\mathrm{C}\left(\mathrm{e}_{2}, \mathrm{f}, \mathrm{m}\right)\right)$.

If $m=m_{0}$ we can argue as above except for $x=\alpha\left(e_{2}, f, m_{0}\right)$. 
Then $D\left(x, m_{0}\right)=C\left(e_{1}, m_{0}, f, n\right)$, which is in $S_{w f}$ by the induction hypothesis. This shows that $C(e, f, n) \in S_{w f}$.

Moreover, totality of $\alpha(e, f, n)$ is equivalent to totality of $\alpha\left(e_{1}, m_{0}, f, n\right)$ in $I\left(C\left(e_{1}, m_{0}, f, n\right)\right)$ which again is equivalent to $n=$ $\mathrm{n}_{0}$.

This proves a) for case $\mathrm{S} 4$.

To prove $b$ ), recall that the extending chains in a products are based on an extension $\mathrm{A}$ in the parameter-space $\mathrm{X}$ and chains in the corresponding $\mathrm{Y}_{\mathrm{A}}$. Since $\alpha(\mathrm{e}, \mathrm{f}, \mathrm{n})$ is total except at most at $\left(\mathrm{m}_{0}, \alpha\left(\mathrm{e}_{2}, \mathrm{f}, \mathrm{m}_{0}\right)\right)$, and since $\alpha\left(e_{2}, f, m_{0}\right)$ is not an extension it follows that $\alpha(e, f, n)$ will meet all extending chains, and $b$ ) follows.

To prove $c$ ), notice that an extension in a product space will send all total elements to extensions. Since $\alpha\left(e_{1}, m_{0}, f, n\right)$ is not an extension, $\left.c\right)$ follows.

S8:

$$
\{e\}(f)={ }^{3} E\left(\lambda g .\left\{e_{1}\right\}(g, f) .\right.
$$

For $n \neq 0,1$ the case is trivial.

8.0: $C(e, f, 0)$ represents $\Pi(g \in \mathcal{N} \rightarrow \mathcal{N}) I\left(C\left(e_{1}, g, f, 0\right)\right.$, so $C(e, f, 0)$ will be in $S_{w f}$ independent of the values of $\left\{e_{1}\right\}(g, f)$ for various $g$. If $\left\{e_{1}\right\}(g, f)=0$ for all $g$, then $\alpha(e, f, 0)$ representing $\lambda g . \alpha\left(e_{1}, g, f, 0\right)$ is total, otherwise not.

In any case, $\alpha(e, f, 0)$ is not an extension because $\alpha(e, f, 0)(g)$ is not an extension for any $g$.

Moreover $h_{\alpha(e, f, 0), C(e, f, 0)}$ is total because $h_{\alpha\left(e_{1}, g, f, 0\right), C\left(e_{1}, g, f, 0\right)}$ are total for all $\mathrm{g}$.

8.1: $C(e, f, 1)$ represents $I(C(e, f, 0)) \rightarrow \mathcal{N}$ and is in $S_{w f}$. We have

$$
\begin{aligned}
& \{e\}(f)=1 \\
\Leftrightarrow & \alpha(e, f, 0) \text { is not total in } I(C(e, f, 0)) \\
\Leftrightarrow & \alpha(e, f, 1) \text { is total in } I(C(e, f, 1)) .
\end{aligned}
$$

This shows a), and b) and c) are trivial in this case.

This ends the proof of the lemma and of Theorem 4.1. 


\section{REFERENCES}

1. Berger, U.: Total sets and objects in Domain Theory, Submitted for publication.

2. Girard, J.-Y.: Une extension de l'interprétation de Gödel et à la théorie des types etc., in J. E. Fenstad ( ed. ): Proc. 2nd Scand. Log. Symp. , North Holland ( 1971 )

3 Girard, J.-Y.: $\Pi_{2}^{1}$-logic ,Part 1: Dilators, Ann. Math Logic 21, pp 75-219, 1981.

4 Girard, J.-Y.: Proof theory and logical complexity, part II, to appear at editions North Holland.

5. Girard, J.-Y.: The system F of variable types fifteen years later, TCS 45 ( 1986 )

6. Kleene, S. C.: Recursive functionals and Quantifiers of finite types I, T.A.M.S. 91 ( 1959 ), 1 - 52.

7. Kristiansen, L.: Thesis, in preparation.

8. Normann, D.: Formalizing the notion of total information, in P. P. Petkov( ed. ) Mathematical Logic, Plenum Press ( 1990 ) 67-94

9. Normann, D. : Wellfounded and non-wellfounded types of continuous functionals, Oslo Preprint Series in Mathematics No 6 ( 1992 ) 
